\title{
HADAMARD GAP SERIES AND NORMAL FUNCTIONS
}

\section{R. SONS AND D. M. CAMPBELL}

In this note we prove the following theorem.

THEOREM 1. Let $f$ be an analytic function defined in the unit disc $\{z|| z \mid<1\}$ by $f(z)=\sum_{k=0}^{x} c_{k} z^{m k}$ where $n_{k+1} / n_{k} \geqslant q>1$. Then $f$ is normal if and only if $f$ is Bloch if and only if lim sup $\left|c_{k}\right|<\infty$.

J. S. Hwang [3] has proved the above theorem in the special case where $c_{k}=n_{k}^{m}$ for some $m>1$ and $\left(n_{k+1} / n_{k}\right) \rightarrow \infty$ as $k \rightarrow \infty$.

The proof of Theorem 1 is based on ideas of $\mathrm{W}$. Fuchs and techniques which go back to Hardy and Littlewood. Throughout the remainder of the paper $p_{0}$ will denote the maximum of $2 \log 20 /(q-1-\log q)$ and $\log 10 /\left(\log q-1+q^{-1}\right)$.

Consider the associated real valued function $F$ defined by

$$
F(x)=\sum_{n=0}^{\infty} x^{n_{k}}, \quad n_{k+1} / n_{k} q>1 .
$$

By accentuating the dominance of the largest term of this series by successive differentiations Fuchs [2] proved for all integers $p$ with $p \geqslant p_{0}$ that $F^{(p)}(x)$ behaves in certain intervals like a single term. To be precise, if $p \geqslant p_{0}$ and $v$ is sufficiently large, then for any $s$ in the interval

$$
\exp \left(\frac{-p}{n_{v}}\right) \leqslant s \leqslant \exp \left(\frac{-p}{2 n_{\mathrm{r}}}\left(1+\frac{\log q}{q-1}\right)\right)
$$

we have the inequality

$$
\sum_{k \neq r} n_{k}\left(n_{k}-1\right) \ldots\left(n_{k}-p+1\right) s^{n_{k}} \leqslant 1 / 4 n_{t}\left(n_{\mathrm{r}}-1\right) \ldots\left(n_{v}-p+1\right) s^{n^{\prime}} .
$$

This observation about the associated real valued function $F$ lets us prove the following important result.

THEOREM 2. Let $f$ be an analytic function defined in $\{z|| z \mid<1\}$ by $f(z)=\sum c_{k} z^{m,}$, $n_{k+1} / n_{k} \geqslant q>1$, where $\lim \sup \left|c_{k}\right|=\infty$. Then for all integers $p$ with $p \geqslant p_{0}$ and all $M>0$, there is an s such that

$$
(1-|z|)^{p}\left|f^{(p)}(z)\right| \geqslant M
$$

for every $z$ on the circle $|z|=s$.

Received 16 January 1979; revised 9 April 1979. 
Proof. Let $\mu(r)=\sup _{k}\left|c_{k}\right| r^{n_{k}}$. The analyticity of $f$ in $|z|<1$ and $\lim \sup \left|c_{k}\right|=\infty$ together imply that $\mu(r)$ is a monotone increasing function which tends to infinity as $r \rightarrow 1$.

Let $p$ be an arbitrary integer greater than $p_{0}$. Choose $r_{0}$ such that $\mu\left(r_{0}\right) \geqslant 4 M(2 e)^{p} / p$ !. Let $v$ be the largest integer such that

$$
\left|c_{v}\right| r_{0}^{n_{r}} \geqslant \mu\left(r_{0}\right) / 2 \text {. }
$$

We note that $r_{0}$ can be chosen near enough to 1 so that $n_{v} \geqslant p$, and we assume henceforth that $r_{0}$ is so chosen. Now set $s=s_{0} r_{0}$ where $s_{0}=\exp \left(-p / n_{\imath}\right)$. Then

$$
\left|f^{(p)}\left(s e^{i q}\right)\right| \geqslant n_{v}\left(n_{v}-1\right) \ldots\left(n_{v}-p+1\right)\left|c_{v}\right| s^{n_{v}-p}-\sum_{k \neq v} n_{k}\left(n_{k}-1\right) \ldots\left(n_{k}-p+1\right)\left|c_{k}\right| s^{n_{k}-p}
$$

Using (1) and (2) we obtain

$$
\begin{aligned}
& \sum_{k \neq r} n_{k}\left(n_{k}-1\right) \ldots\left(n_{k}-p+1\right)\left|c_{k}\right|\left(s_{0} r_{0}\right)^{n_{k}-p} \\
& \quad \leqslant \sup _{k}\left(\left|c_{k}\right| r_{0}^{n_{k}}\right) r_{0}^{-p} s_{0}^{-p} \sum_{k \neq v} n_{k}\left(n_{k}-1\right) \ldots\left(n_{k}-p+1\right) s_{0}^{n_{k}} \\
& \leqslant 1 / 2 n_{v}\left(n_{v}-1\right) \ldots\left(n_{v}-p+1\right)\left|c_{v}\right| s_{0}^{n_{v}-p} r_{0}^{n_{v}-p} .
\end{aligned}
$$

Thus from (3) and (4) we find

$$
\begin{aligned}
\left|f^{(p)}\left(s e^{i \theta}\right)\right| & \geqslant 1 / 2 n_{v}\left(n_{v}-1\right) \ldots\left(n_{v}-p+1\right) s_{0}^{n_{r}-p} r_{0}^{n_{r}-p}\left|c_{v}\right| \\
& \geqslant n_{v}\left(n_{v}-1\right) \ldots\left(n_{v}-p+1\right) M 2^{p} / p !
\end{aligned}
$$

And therefore

$$
\begin{aligned}
(1-s)^{p}\left|f^{(p)}\left(s e^{i \theta}\right)\right| & \geqslant\left(1-s_{0}\right)^{p} n_{v}\left(n_{v}-1\right) \ldots\left(n_{c}-p+1\right) M 2^{p} / p ! \\
& \geqslant\left(\frac{p}{n_{t}}\right)^{p} n_{v}\left(n_{v}-1\right) \ldots\left(n_{t}-p+1\right) M / p ! \\
& =\frac{M p^{p}}{p !}\left(1-\frac{1}{n_{t}}\right)\left(1-\frac{2}{n_{t}}\right) \ldots\left(1-\frac{p-1}{n_{v}}\right) \\
& \geqslant \frac{M p^{p}}{p !}\left(1-\frac{1}{p}\right)\left(1-\frac{2}{p}\right) \ldots\left(1-\frac{p-1}{p}\right) \equiv M,
\end{aligned}
$$

which concludes the proof of Theorem 2 .

Proof of Theorem 1. Pommerenke [8] proved lim sup $\left|c_{k}\right|<\infty$ implies $f$ is Bloch, and it is well known that Bloch functions are normal [9, p. 268]. It therefore suffices to prove that $\lim \sup \left|c_{k}\right|=\infty$ implies $f$ is not normal.

Fix an integer $p$ for which $p>p_{0}$. Lappan [4] proved that if $f$ is normal, then there is a finite constant $K$ such that

$$
\frac{\left|f^{(p)}(z)\right|(1-|z|)^{p}}{1+|f(z)|^{p+1}} \leqslant K
$$


for all $z$ in the unit disc. According to Theorem 2 there is a sequence of radii $s_{n}$ such that for $|z|=s_{n}$

$$
(1-|z|)^{p}\left|f^{(p)}(z)\right| \geqslant n
$$

If $\min |f(z)|$ on $|z|=s_{n}$ tends to $\infty$, then $f$ has Koebe arcs and is therefore non-normal $[9, \mathrm{p} .267]$. If $\min |f(z)|$ on $|z|=s_{n}$ does not tend to $\infty$, then by passing to a subsequence we can find an integer $M$ and a sequence of points $z_{n},\left|z_{n}\right|=s_{n}$, such that $\left|f\left(z_{n}\right)\right| \leqslant M<\infty$. For this sequence of points

$$
\frac{\left(1-\left|z_{n}\right|\right)^{p}\left|f^{(p)}\left(z_{n}\right)\right|}{1+\left|f\left(z_{n}\right)\right|^{p+1}} \geqslant \frac{n}{1+M^{p+1}},
$$

which proves that (6) cannot hold. Therefore $f$ must be non-normal.

Corollary. Let $f$ be defined in the unit disc by $f(z)=\sum_{k=1}^{\infty} k^{k} z^{2^{k}}$. Then all of its derivatives and all of its integrals are non-normal functions which are analytic in $\{z|| z \mid<1\}$.

Remark 1. Motivated by a result of MacLane [5, p. 46], Bonar [1, p. 59] posed the following question. If $f$ is a strongly annular function, can $f$ be written as $f(z)=g(z)+h(z)$ where $g(z)=\sum a_{k} z^{\mu_{k}}, \lim \inf \mu_{k+1} / \mu_{k}>3$, and $h(z)$ is bounded in the unit disc? The answer is no. To see this, let $F(z)=\sum 2^{k} z^{2 k}$. Since $n_{k+1} / n_{k}=2$, and $\left|c_{k}\right| \rightarrow \infty$, it is an easy consequence of Theorem 2 that for an integer $p>p_{0}$, there is an increasing sequence of positive numbers $s_{n}$ such that $(1-|z|)^{p}\left|F^{(p)}(z)\right| \geqslant n$ for all $z$ on the circle $|z|=s_{n}$. Thus $F^{(p)}$ is strongly annular. Suppose $F^{(p)}(z)$ could be written as $g(z)+h(z)$ where $g(z)=\sum a_{j} z^{\mu_{j}}, \lim \inf \mu_{j+1} / \mu_{j}>3$, and $h$ is bounded in the unit disc. The coefficients in the power series expansion of $h$ about zero must go to zero since $h$ is bounded. Therefore for $k$ sufficiently large the index set $\left\{\mu_{j}\right\}$ must contain all exponents of the form $\left\{2^{j}-p\right\}_{j=k}^{\alpha}$. Consequently, $3<\liminf \mu_{j+1} / \mu_{j} \leqslant 2$, which is absurd. An appropriate modification works for any $q>1$.

Remark 2. Piranian [7] asked whether a bounded function of finite area must have a normal derivative. Theorem 1 lets us answer this in the negative. Let $f$ be defined by

$$
f(z)=\sum_{n=1}^{\infty} n\left(2^{n}+1\right)^{-1} z^{2 n+1}=\sum_{j=1}^{\infty} a_{j} z^{j} .
$$

Then $f$ is bounded by $\sum n 2^{-n}$, and $\sum j\left|a_{j}\right|^{2} \leqslant \sum n^{2} 2^{-n}$ shows that $f$ has finite area. Theorem 1 guarantees that $f^{\prime}(z)=\sum n z^{2 \prime}$ defines a non-normal function in the unit disc.

We close with two open questions.

Question 1. If $f(z)=\sum c_{k} z^{m_{k}}, \quad n_{k+1} / n_{k} \geqslant q>1, \lim \sup \left|c_{k}\right|=x$, must $f$ be annular? If the maximum modulus of $f$ grows rapidly enough, the answer is yes [c.f. 6 , Thm. 4].

Question 2. What is the best value for $p_{0}$ for which Theorem 2 is true? 


\section{References}

1. D. D. Bonar, On annular functions (VEB Deutscher Verlag Wiss., 1971).

2. W. Fuchs, "On the zeros of power series with Hadamard gaps", Nagoya Math. J., 29 (1967), 167-174.

3. J. S. Hwang, "Plessner points, Julia points, and $\rho^{*}$-points", J. Math. Kyoto, 18 (1978), 173-188.

4. P. Lappan, "The spherical derivative and normal functions", Ann. Acad. Sci. Fenn. Ser AI, 3 (1977), 301310.

5. G. R. MacLane, “Asymptotic values of holomorphic functions”, Rice University Studies, 49 (1963), 1-83.

6. P. J. Nicholls and L. R. Sons, "Minimum modulus and zeros of functions in the unit disc", Proc. London Math. Soc. (3), 31 (1975), 99-113.

7. G. Piranian, "Univalence and the spherical second derivative" (preprint).

8. Ch. Pommerenke, "On Bloch functions", J. London Math. Soc. (2), 2 (1970), 689-695.

9. Ch. Pommerenke, Univalent functions (Vandenhoeck and Ruprecht, 1975).

\section{Mathematics Sciences Department, Northern Illinois University, DeKalb, Ill, 60115, U.S.A.}

\section{Mathematics Department,} University of Michigan, Ann Arbor, Mich. 48109, U.S.A. 